\title{
Non-constructive Supracardiac Total Anomalous Pulmonary Venous Connection With Giant Superior Vena Cava Aneurysm
}

\author{
Yangbo Yan $^{1}$, Kai Yu ${ }^{1}$, Yanlin Yang $^{1}$, and Changping Gan ${ }^{1}$ \\ ${ }^{1}$ Sichuan University West China Hospital
}

November 27, 2020

\begin{abstract}
Giant superior vena cava (SVC) aneurysm in obstructive supracardiac total anomalous venous connection (TAPVC) is rare, and there has not been a giant SVC aneurysm reported in non-obstructive TAPVC. Here we reported a 29-year-old female with non-obstructive TAPVC and a giant SVC aneurysm. Routine TAPVC correction and partial venoctomy were done, considering that such a giant aneurysm in a non-obstructive venous arch might have its histological etiology and higher risk of thrombosis and/or rupture.
\end{abstract}

Title page

Non-constructive Supracardiac Total Anomalous Pulmonary Venous Connection With Giant Superior Vena Cava Aneurysm

Yangbo Yan $\mathrm{MS}^{1}$,Kai Yu MS ${ }^{1}$, Yanlin Yang $\mathrm{MS}^{1}$, Changping Gan MD, $\mathrm{PhD}^{1,2}$

${ }^{1}$ Department of Cardiovascular Surgery, West China Hospital, Sichuan University, No.37 Guo Xue Alley, Chengdu 610041, China

${ }^{2}$ Corresponding to: Dr. Changping Gan, MD, Department of Cardiovascular Surgery, West China Hospital, Sichuan University, 37\# Guoxue Alley, Chengdu, 610041, China.

Email: ganchangping@hotmail.com

Data availability statement: Data sharing is not applicable to this article as no new data were created or analyzed in this study.

Funding statement: This study was partially supported by grants from the Science and Technology Department of Sichuan Province (2020YJ0239) and the Health Planning Committee of Sichuan Province (20PJ052). The funders had no role in the study design, data collection and analysis, writing of the manuscript, and decision to submit the article for publication.

Conflict of interest: None.

Institutional Review Board approval: Institutional review board approval of our hospital was obtained for this study.

Patient consent statement: The patient involved in this study has given her informed consent.

\section{Manuscript}

Abstract: Giant superior vena cava (SVC) aneurysm in obstructive supracardiac total anomalous venous connection (TAPVC) is rare, and there has not been a giant SVC aneurysm reported in non-obstructive TAPVC. Here we reported a 29-year-old female with non-obstructive TAPVC and a giant SVC aneurysm. 
Routine TAPVC correction and partial venoctomy were done, considering that such a giant aneurysm in a non-obstructive venous arch might have its histological etiology and higher risk of thrombosis and/or rupture.

Key words: total anomalous pulmonary venous connection, superior vena cava, aneurysm

Giant superior vena cava (SVC) aneurysm in obstructive supracardiac total anomalous venous connection (TAPVC) is rare ${ }^{(1-3)}$, and there has not been a giant SVC aneurysm reported in non-obstructive TAPVC. Here we reported a 29-year-old female with non-obstructive TAPVC and a giant SVC aneurysm.

The patient was seen in the clinic due to her dyspnea and impaired exercise tolerance. Preoperative echocardiography and computed tomography (CT) confirmed the diagnosis of supracardiac TAPVC with a giant venous arch complicated with a $22 \mathrm{~mm}$ atrial septal defect (ASD) (Figure 1A-1B;supplementary material online, video 1). The maximum diameter of the SVC was measured $7.68 \times 6.32 \mathrm{~cm}$ (Figure 1C). Neither the echocardiography nor the CT found any obstruction throughout the venous pathway. Angiography was conducted which showed a mean pulmonary artery pressure of $22 \mathrm{mmHg}$ and low pulmonary resistance. Intraoperative findings were consistent with all preoperative diagnosis (Figure 1D). While establishing the cardiopulmonary bypass, along with the routine IVC cannulation, two separate venous catheters were placed into the right brachiocephalic vein and the innominate vein respectively. Routine TAPVC correction was done by anastomosing the posterior wall of left atrium with the pulmonary venous confluence, ligating the vertical vein and repairing the ASD.

Although some publications concluded that, comparing with a saccular shape, a fusiform SVC aneurysm will usually have a benign prognosis ${ }^{(4)}$. However, considering that such a giant SVC aneurysm presented in a non-obstructive environment, we thought it might have its histological etiology and would take a higher risk of thrombosis and/or rupture. Therefore, a partial venoctomy was reasonable. By controlling the distal veins, the wall of the aneurysm was partially resected, and the diameter of SVC was reduced to a normal size (Figure 1E). Pathology of the SVC revealed desmoplasia with hyaline degeneration and calcification in the vascular wall and mucoid degeneration in the focal area. The patient had an uncomplicated postoperative course and was discharged on the seventh postoperative day.

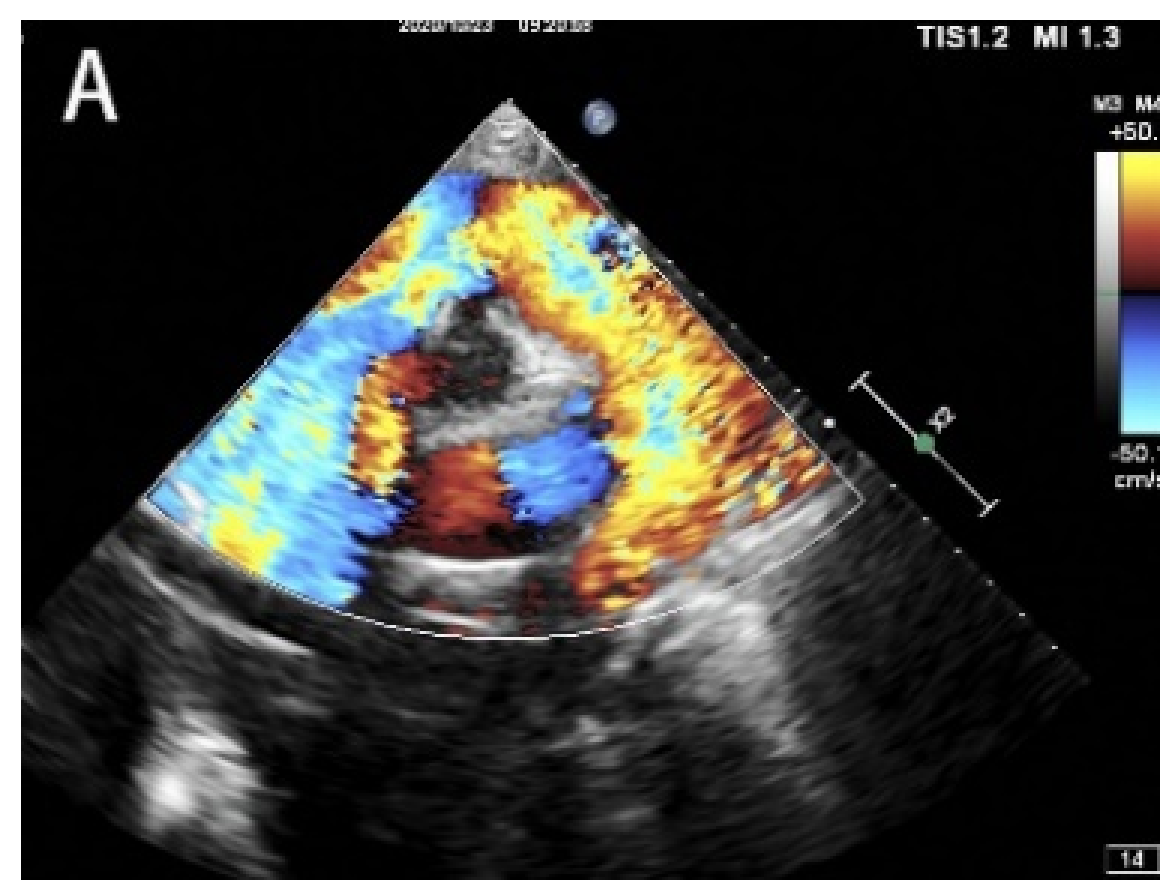




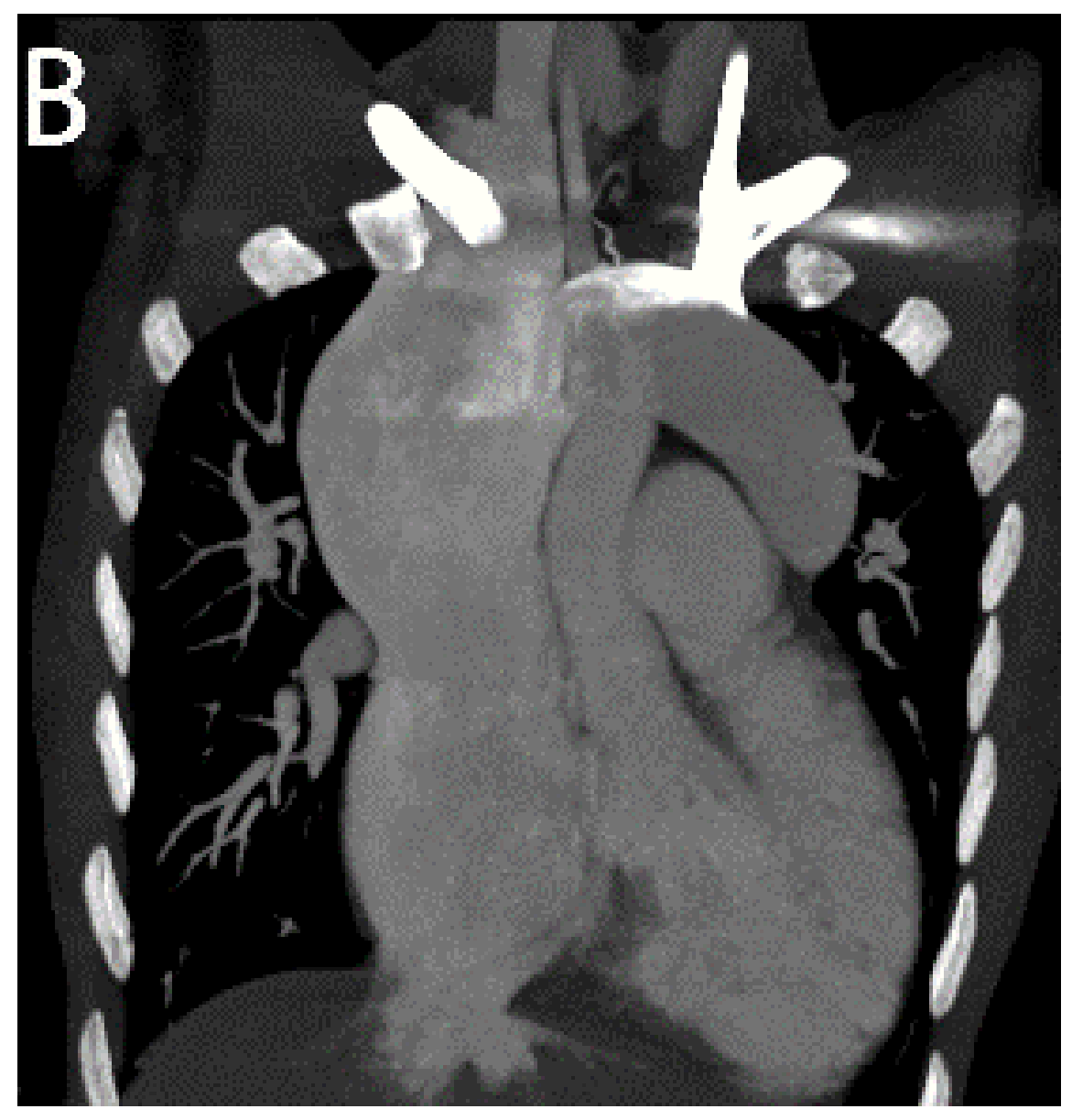




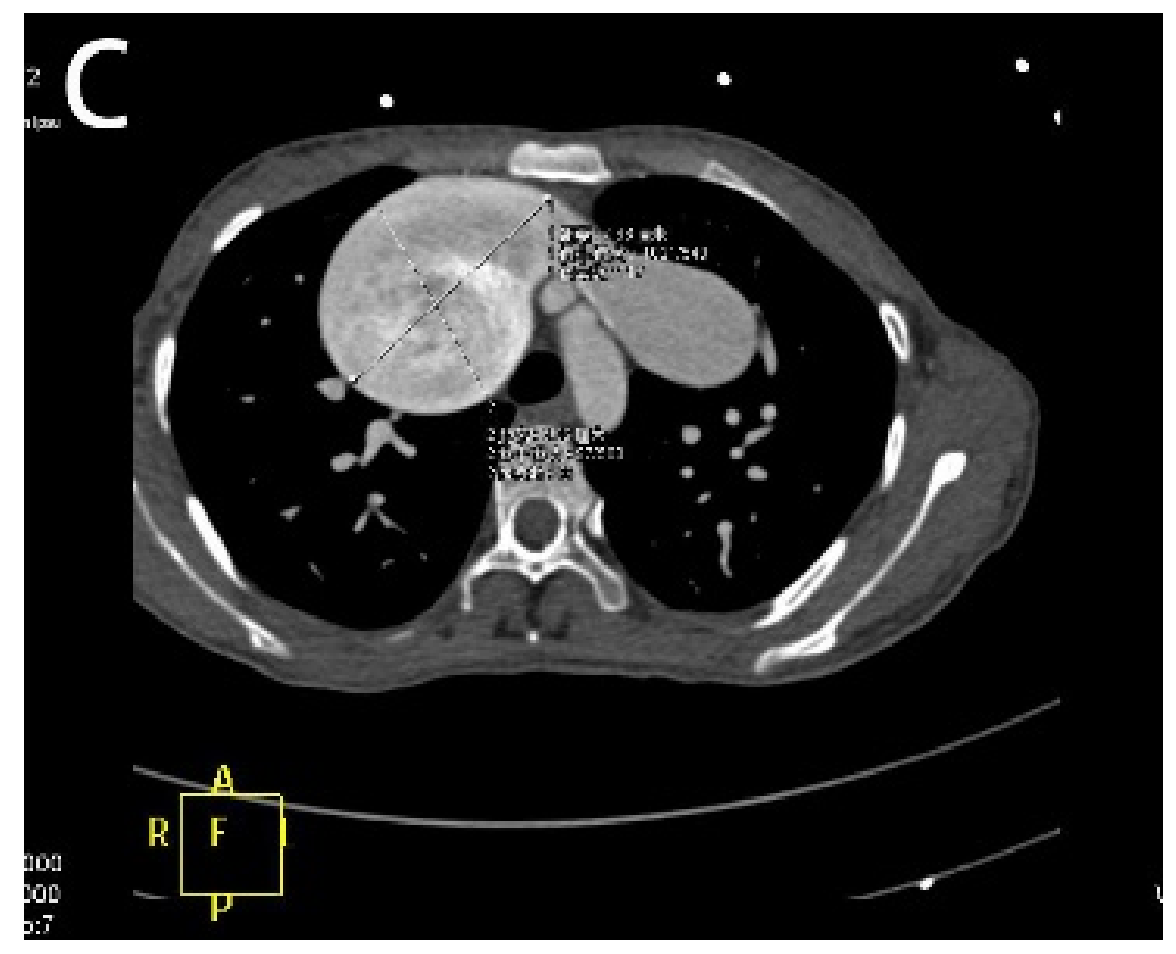




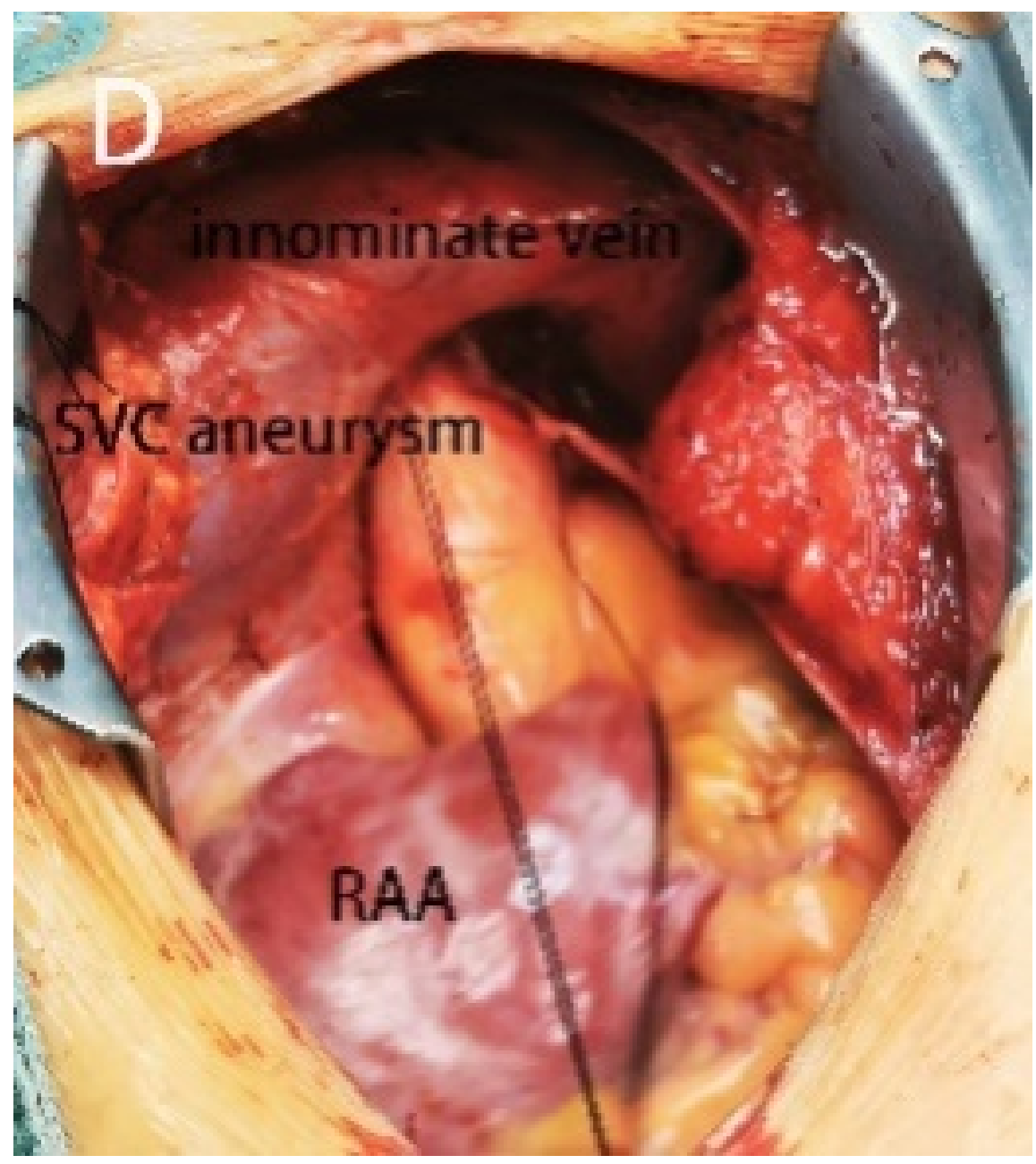




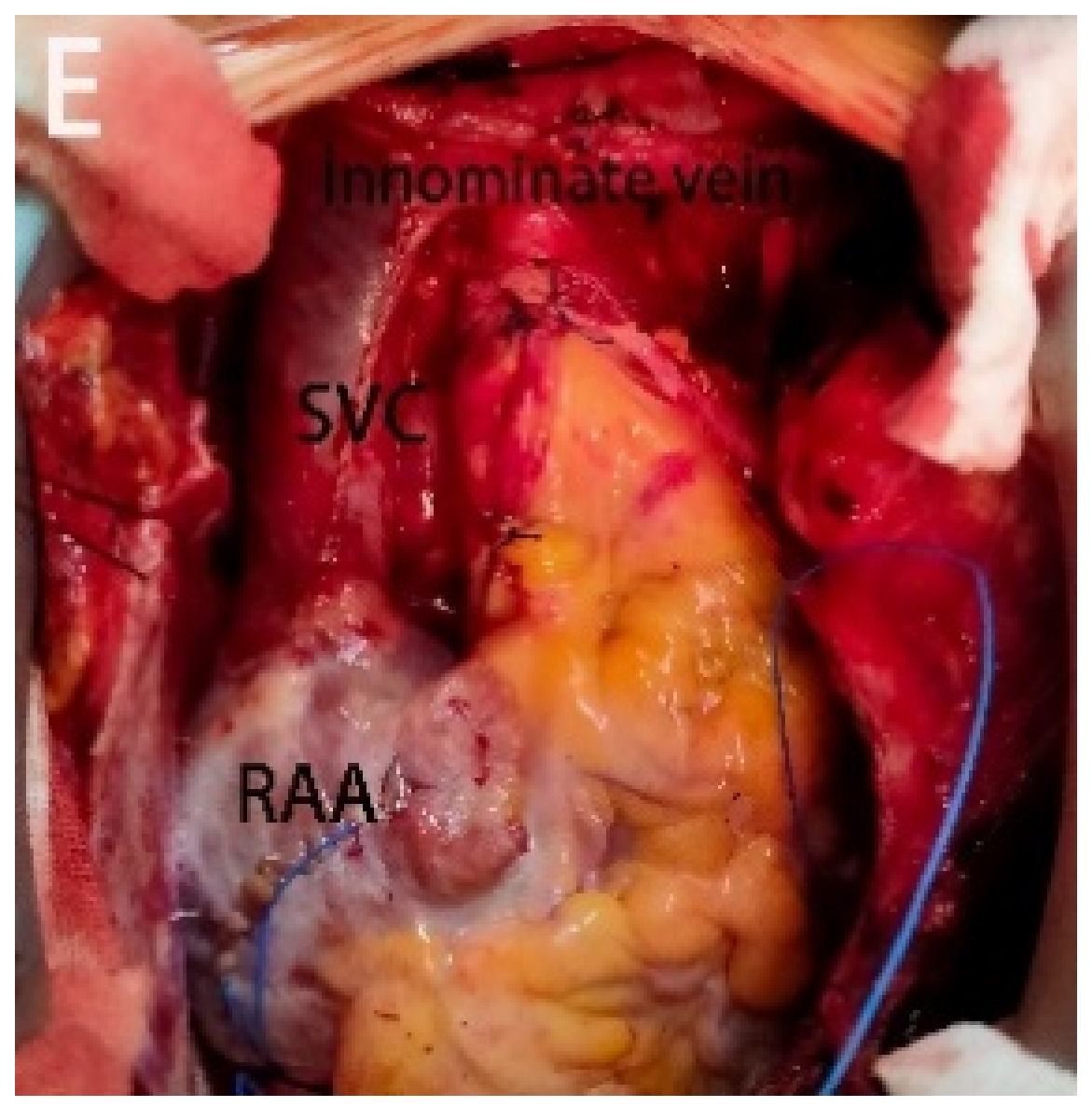

FIGURE 1:1A-B Preoperative transthoracic echocardiography and CT showed supracardiac TAPVC with a giant venous arch. C CT showed the maximum diameter of the SVC. D-E Pre and postoperative view of SVC. CT, computed tomography; TAPVC, total anomalous

pulmonary venous connection; SVC, superior vena cava; RAA, right atrial appendage.

\section{Reference}

1. Vida VL, Leon-Wyss J, Veras O, Vides I, Castaneda AR. Rare case of obstructive supracardiac total anomalous pulmonary venous connection in association with a giant superior vena cava. Ann Thorac Surg 2006; 81(3): 1141.

2. Tsai YS, Yu CH, Pang KK, Shih SL. A giant superior venous cava resulted from obstructive total anomalous pulmonary venous connection. Circ Cardiovasc Imaging 2009; 2(2): 161-2.

3. Thummar AC, Phadke MS, Lanjewar CP, Nabar AA, Kerkar PG. Supracardiac total anomalous pulmonary venous drainage with giant superior vena cava aneurysm: a rare combination. J Am Coll Cardiol 2014; 63(19): e51.

4. Koga S, Ikeda S, Sanuki Y, Ninomiya A, Izumikawa T, Miyahara Y, Kohno S. A case of asymptomatic fusiform aneurysm of the superior vena cava detected by magnetic resonance imaging. Int J Cardiol 2006; 113(2): e39-41.

5. Oh SG, Kim KH, Seon HJ, Yoon HJ, Ahn K, Jeong MH, Cho JG, Park JC, Kang JC. Unusual cause of acute right ventricular dysfunction: rapid progression of superior vena cava aneurysm complicated by 
thrombosis and pulmonary thromboembolism. J Korean Med Sci 2011; 26(5): 690-3

6. Panduranga P, Thomas E, Al-Maskari S, Al-Farqani A. Giant superior vena caval aneurysm in a postGlenn patient. Interact Cardiovasc Thorac Surg 2012; 14(6): 878-9.

7. Varma P, Dharan B, Ramachandran P, Neelakandhan K. Superior vena caval aneurysm. Interactive Cardiovascular and Thoracic Surgery 2003; 2(3): 331-3. 